\title{
Concept study of radar sensors for near-field tsunami early warning
}

\author{
T. Börner ${ }^{1}$, M. Galletti ${ }^{1, *}$, N. P. Marquart ${ }^{1,{ }^{* *}}$, and G. Krieger ${ }^{1}$ \\ ${ }^{1}$ Microwaves and Radar Institute, German Aerospace Center in the Helmholtz Alliance (DLR), Oberpfaffenhofen, Germany \\ *now at: NOAA, Norman, OK, USA \\ ${ }^{* *}$ now at: European Patent Office, Munich, Germany
}

Received: 16 September 2009 - Revised: 9 September 2010 - Accepted: 10 September 2010 - Published: 21 September 2010

\begin{abstract}
Off-shore detection of tsunami waves is a critical component of an effective tsunami early warning system (TEWS). Even more critical is the off-shore detection of local tsunamis, namely tsunamis that strike coastal areas within minutes after generation. In this paper we propose new concepts for near-field tsunami early detection, based on innovative and up-to-date microwave remote sensing techniques. We particularly introduce the NESTRAD (NEar-Space Tsunami RADar) concept, which consists of a real aperture radar accommodated inside a stationary stratospheric airship providing continuous monitoring of tsunamigenic oceanic trenches.
\end{abstract}

\section{Introduction}

In the first few minutes after an earthquake conventional tsunami warning systems mainly rely on a number of seismic estimates of the earthquake, e.g. magnitude, focal depth, location, to determine whether a tsunami might have been generated with a certain probability. These measures are usually available after a short time $(<10 \mathrm{~min})$, only limited by the time it takes for the seismic waves to travel from the epicentre to the seismic stations. However, at this early stage the tsunami warning system does not yet know whether a tsunami has really been generated or not.

Far-field tsunamis, which hit a coastline after a sufficiently large amount of time, can easily be detected and measured using conventional techniques like tide gauges and buoys in combination with seismic results. The warning can then be

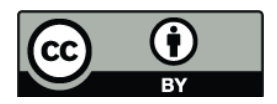

Correspondence to: $\mathrm{T}$. Börner

(thomas.boerner@dlr.de) issued together with a good knowledge of the direction and size of the tsunami wave, and false alarms are merely nonexistent.

When dealing with near-field tsunamis, it might be sufficient to issue warnings for all tsunamigenic earthquakes because of tight time constraints, but repeated false-alarms can lead to a reduced responsiveness of the population to such warnings, which should be avoided for obvious reasons. In order to bring useful information to existing warning systems (i.e. has a tsunami been generated or not, velocity, direction, etc.) space-based sensors must hence provide responses within a few minutes from the initial quake, because the wave might hit the coast within e.g. 20-30 min when it is generated at the Sunda trench and approaching the Indonesian coast. Late information is of little value to early-warning, since cheaper, ground-based technologies (e.g. tide gauges or tsunami buoys) have most probably already provided a direct and precise measurement. Early information, however, can only be collected in the area where the tsunami is generated, i.e. in the open ocean, where the wave height and the orbital wave velocities are very low compared to the wave parameters near or at the coast (see Sect. 2). Initial seismic measures are of course required as input to such space-based sensors, particularly the location of the epicentre, in order to point the sensor to the potential tsunami wave as quick as possible. To match these constraints, a careful analysis of the limitations in terms of time-responsiveness of LEO (low earth orbit) satellites and the opportunities offered by different space and airborne platforms is necessary.

Stratospheric airships are an interesting solution for services demanding continuous temporal coverage and continuous data downlink. Stratospheric airships are unmanned, autonomous solar-powered vehicles designed to operate above the jet-stream at approximately $20 \mathrm{~km}$ and to carry payloads

Published by Copernicus Publications on behalf of the European Geosciences Union. 
of up to a few tons (Hanlon, 2005; Qureshi, 2003; Cooney, 2009). The airships, measuring more than $100 \mathrm{~m}$ in length, can be used for both military and civilian applications. Besides early-warning and post-disaster damage assessment, they offer reliable and continuous downlink transmission and might also serve the purpose of mobile data communication.

The NESTRAD concept presented in this paper consists of a C-band phased-array radar accommodated inside a stratospheric airship, providing quasi-geostationary, all-weather, $24 / 7$ coverage and consequently a response time less than $5 \mathrm{~min}$. It is meant to detect tsunami-induced RCS modulations (tsunami shadows) (Godin, 2004; Troitskaya et al., 2008), tsunami wave height as well as tsunami orbital velocities. Depending whether the platform is stationary or slowmoving, it can operate as a real aperture radar or as a synthetic aperture radar (SAR).

The performance analysis shown in the following tries to give an account on the system capabilities to resolve tsunamirelated geophysical features. In particular, the detection of radar cross section modulations (Godin, 2004) is expected to provide wide area coverage. In this case modulations of a few $\mathrm{dB}$ should be present for tsunamis of virtually any size.

\section{Radar relevant tsunami signatures}

In this section we review, from a radar remote sensing viewpoint, all known tsunami-related signatures. For each detectable geophysical feature, every sensor with either proven or potential detection capability is listed and briefly discussed. Each sensor relies on at least one principle of detection but, obviously, one sensor can combine more principles of detection.

\subsection{Tsunami wave height}

Wave height is the most intuitive physical feature of an ocean wave and can be measured by tide gauges at shore, ocean bottom pressure sensors off shore and, lately, tsunami wave height measurements were recorded by satellite-borne altimeters (e.g. Okal, 1999). Satellite altimetry relies on nadirpointing radars carried onboard a number of satellite missions like ESA's ERS-1, ERS-2, ENVISAT, SENTINEL and the series of US-French satellites Topex/Poseidon, Jason-1 and Jason-2. On Boxing Day 2004, a number of altimeters accidentally overflew the tsunami wave and provided the scientific community with valuable measurements (e.g. a wave amplitude of $0.7 \mathrm{~m}$ in the open ocean). Following this accidental data-takes, concepts were put forth for tsunami earlywarning from space. The proposed concepts envision a number of LEO microsatellites carrying altimeters (implemented either with active or passive radar technology). For example, results from the GANDER project (Global Altimeter Network Designed to Evaluate Risk) (Allen, 2006) put forth a constellation of 24 micro-satellites (4 satellites in each of 6 near-polar orbits at $1682 \mathrm{~km}$ altitude, orbit period $2 \mathrm{~h}$ ) in order to overfly a tsunami within $30 \mathrm{~min}$ from the initial quake. An interesting alternative to classical altimeters is to use GNSS reflectometry, as proposed in (Stosius, 2010). However, the satellite still has to overfly a ground station for the data downlink, which might well take another 30 min under rather favourable conditions. If a constellation of satellites is put forth for tsunami early warning, intra-satellite communication links should be envisioned to match the constraints dictated by near-field tsunami early warning.

Since tsunami wave heights in the open ocean are expected to be far below $1 \mathrm{~m}$, detecting such a small change will certainly be a challenge. The Topex/Poseidon and Jason-1/2 altimeter measurements already showed that averaging over long times is required to resolve these changes, and it might become impossible for low amplitude tsunamis, especially under bad weather conditions where the ocean is already very rough.

However, one advantage, which is true for all signatures mentioned in this section, is that tsunamis are extremely large-scale phenomena and that the changes, although being very small, apply for very large areas in the order of hundreds times tens of kilometers or even more. By continuously monitoring these large areas and by adequate averaging and filtering, it should be possible to extract even small changes from the overall statistics.

\subsection{Tsunami orbital velocities}

As with every ocean wave, tsunami propagation relies on the elliptical movement of water masses. Even though the group velocity of the wave is generally high $(700 \mathrm{~km} / \mathrm{h}$ in the open ocean, slows down with shallower bathymetry) the horizontal orbital velocities of water masses accomplishing tsunami propagation are extremely small. The horizontal orbital velocity of a tsunami wave $U_{\mathrm{h}}$ can be calculated using the following formula (e.g. Lautrup, 2005; Myers, 2008; Bryant, 2001)

$U_{\mathrm{h}}=A \sqrt{\frac{g}{D}}$

where $A$ is the wave amplitude, $g$ is the gravitational constant and $D$ is the ocean depth. For the Sumatra Boxing Day tsunami in 2004, which had an amplitude of $0.7 \mathrm{~m}$ in the open ocean with a depth of $4000 \mathrm{~m}$, the horizontal orbital velocity $U_{\mathrm{h}}$ results to $3.5 \mathrm{~cm} / \mathrm{s}$. This value certainly represents the upper bound of expected velocities; smaller (average) tsunamis should be in the range of $1-2 \mathrm{~cm} / \mathrm{s}$. These orbital velocities are amplified by shallow bathymetric features and might reach tens of $\mathrm{cm} / \mathrm{s}$ or even $\mathrm{m} / \mathrm{s}$ in coastal areas. Tsunami orbital velocities might be detected by along-track interferometric SAR systems (Romeiser, 2000) and HF surface wave radars (e.g. Lipa, 2006; Anderson, 2008). 


\subsection{Tsunami shadows}

Tsunami shadows are spatially extended alterations of the radar cross section of the ocean surface. Observations of tsunami shadows, i.e., extended darker strips on the ocean surface along the front of a tsunami wave were first reported by the pilot of an aircraft overflying the 1946 Aleutian tsunami (Perkins, 2004). Later, observations were reported by eye-witnesses located at different points along the shore of the island of Oahu, Hawaii, coinciding with the arrival of a small tsunami triggered by the 1994 Shikotan earthquake. Besides optical observations, tsunami shadows were also observed at microwave frequencies by satellite altimeters overflying the Boxing Day tsunami (Godin, 2004; Troitskaya et al., 2008). This unique data-take confirmed the presence of relevant (a few $\mathrm{dB}$ ) variations of the ocean surface radar cross section (RCS) associated with the tsunami wave front. However, the physical mechanisms causing tsunami shadows are not yet entirely understood. The most likely explanation is that the orbital velocities of the tsunami wave cause modulations of wind velocity over the sea surface and thus cause variations of the intensity of short surface waves.

These observations suggest that satellite altimetry, synthetic aperture radar (SAR), scatterometers and radiometers might have good tsunami detection capabilities. Of particular significance is the fact that the tsunami shadows propagate at a known and very distinct speed, which allows for their unambiguous differentiation from other features on the ocean surface. Therefore these shadows appear to be the most promising detectable signature for the near-field problem and for tsunami detection in the open ocean in general. Further research is however needed to evaluate the time needed for tsunami shadows to build up after the tsunami inception.

\subsection{Tsunami-induced internal waves}

Tsunamis are long gravity waves and, like tides, have the capability of triggering internal waves under given oceanographic conditions. Internal waves generated by the Boxing Day tsunami were recorded by MODIS (Santek, 2007). Even though internal waves appear as radar cross section variations of the ocean surface, it is worth reminding that the generating mechanism and the spatial scale of these features are completely different from tsunami shadows.

All these features are ocean surface physical features, and might be detectable by satellite borne sensors (SAR, satellite altimeters, scatterometers and radiometers). Indeed, a number of mechanisms (either known or unknown) might contribute to tsunami-induced radar cross section modulations and chances are that these effects are strong enough to be used as principles of detection by future tsunami warning systems. This involves not only detection but also an estimate of the tsunami magnitude. Ultimately, a stationary sensor (e.g. NESTRAD) has the possibility to learn normal patterns and detection and/or magnitude estimation can be achieved by comparing pre- and post-quake patterns.

\section{The NESTRAD concept}

NESTRAD consists of a phased array real aperture radar operating at microwave frequencies (e.g. C-band, as chosen for the concept shown in the following) accommodated inside a stratospheric airship. Contrary to a satellite, no stowing and deployment of the antenna is required, prompting the use of low power density active electronically scanned array technology (LPD-AESA). In the following, a planar phased array is considered, but other solutions (conformal arrays or a reflector illuminated by a feed array) could be envisioned to achieve full $360^{\circ}$ azimuth coverage using highly innovative digital beamforming techniques (Krieger, 2008). NESTRAD offers four principles of detection. The first is the detection of tsunami-induced radar cross section modulations (RCS mode). The second is the Doppler retrieval of tsunami orbital velocities (Doppler mode). The third is the retrieval of velocities by using ground moving target indication (GMTI) via along-track interferometry (ATI mode), and the fourth is the retrieval of tsunami wave amplitudes obtained by pointing the beam downwards and detecting relative displacements in sea-surface height (altimeter mode). In the following the first three principles will be analyzed and a performance prediction of the proposed design will be provided.

One important error source that has to be mentioned are stratospheric winds and thus uncertainties in the knowledge of the exact position and trajectory of the platform. The altitude of $20 \mathrm{~km}$ (above the jet streams) has been chosen, since minimal wind conditions are to be expected during most of the time. Winds still can change by up to $50 \mathrm{~m} / \mathrm{s}$ e.g. due to sudden stratospheric warming, but these effects are usually slow and smooth (within 1-2 weeks) and only few of these peaks are to be expected over the year (Modica, 2007). Therefore this error source is assumed to be negligible (quasi-constant wind conditions in short time periods) in the performance estimation for the different modes, at least for a first-order estimate.

Before performance prediction, however, we start with spatial coverage considerations.

\subsection{Spatial coverage}

According to the illumination geometry depicted in Fig. 1, the off-nadir angle $\theta$ can be written as

$\tan (\theta)=\left[\frac{R \sin (\varphi)}{H-R \cos (\varphi)}\right] \rightarrow \theta=\arctan \left[\frac{R \sin (\varphi)}{H-R \cos (\varphi)}\right]$,

where $R$ is the earth radius $(6370 \mathrm{~km}), H$ is the platform height from earth center (i.e. $6370 \mathrm{kM}+20 \mathrm{~km}=6390 \mathrm{~km}$ ) and $\varphi=\eta \theta$, where $\eta$ is the incidence angle. The distance $D_{\text {hor }}$ from nadir to horizon is then derived by

$D_{\text {hor }}=R \varphi$. 


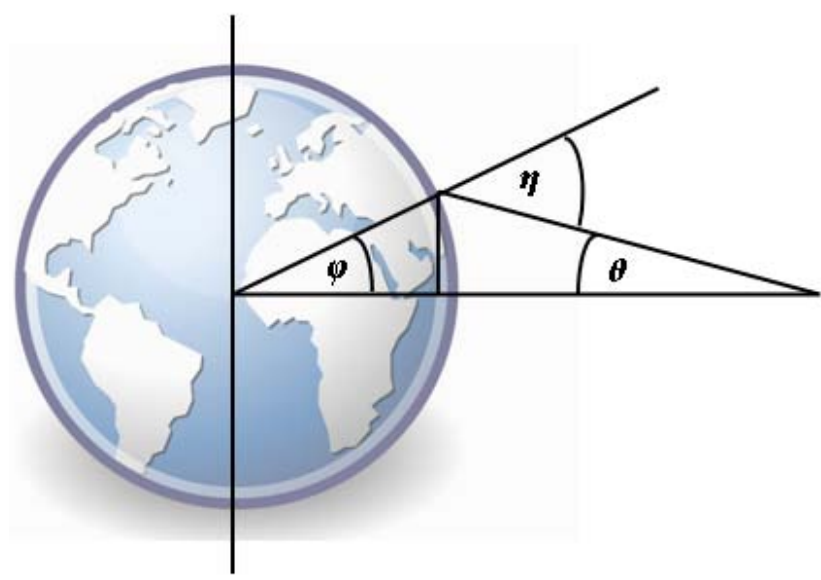

Fig. 1. Sketch of illumination geometry and spatial coverage.

The formulae above account for the geometric horizon (also called visual horizon). At microwave frequencies however, electromagnetic energy is bent slightly downwards. This phenomenon is such that the radar horizon is displaced farther away from the visual horizon. This effect can be taken into account by multiplying the earth radius by $4 / 3$ (provided propagation occurs through a standard atmosphere) (Afullo et al., 2006). For a stratospheric airship located $20 \mathrm{~km}$ above ground, the radar horizon is thus located $575 \mathrm{~km}$ from the nadir point. So, considering the attitude change capabilities of the airship, the radar coverage for target detection is a disk of approximately $1000 \mathrm{~km}$ diameter. For the purpose of tsunami detection however, we must be able to retrieve the RCS of the ocean itself, and not of a target on its surface. In the following section the performance prediction shows acceptable signal-to-noise ratio (SNR) even for $-30 \mathrm{~dB}$ at incidence angles up to $85^{\circ}(250 \mathrm{~km}$ ground range, $500 \mathrm{~km}$ diameter disk), but the variability of ocean surface radar cross section (RCS) at low grazing angles is such that the real monitoring capabilities of the system can only be ascertained by experimental measurements.

In the case of the Sunda trench (see Fig. 2), the length $(3000 \mathrm{~km})$ is such that an array of $4-5$ airships is necessary to provide early warning to the entire Java and Sumatra coastline. The same applies for the Mediterranean Sea (see Fig. 3), where almost every coastline lies in the near-field.

There are however situations where fewer units could cover the whole tsunamigenic areas of concern, providing near-field early warning to densely populated areas like Japan, the Cascadia Subduction Zone (CSZ) off Oregon and British Columbia or the Philippines.

\subsection{RCS (radar cross section) mode}

For the RCS mode we present a performance prediction based on the single pulse radar equation, known in the literature as the SLAR radar equation (Henderson et al., 1998). In

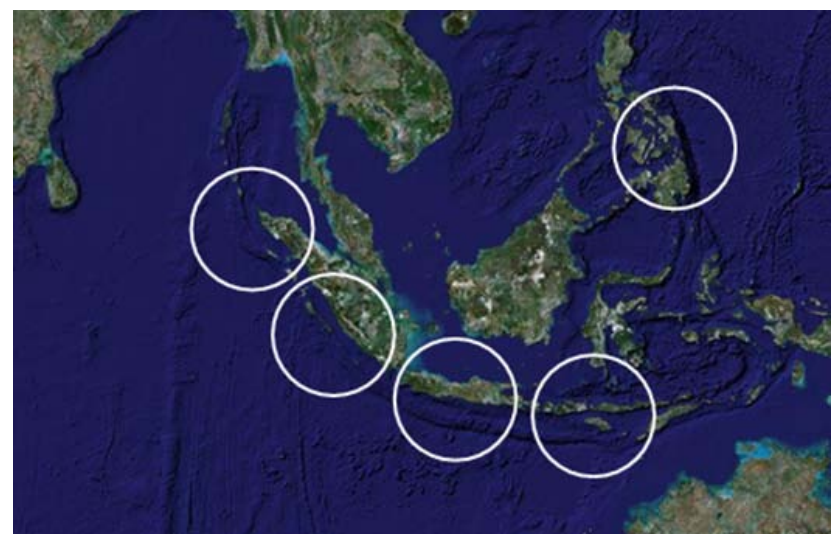

Fig. 2. Spatial coverage for Sunda trench and the Philippines.

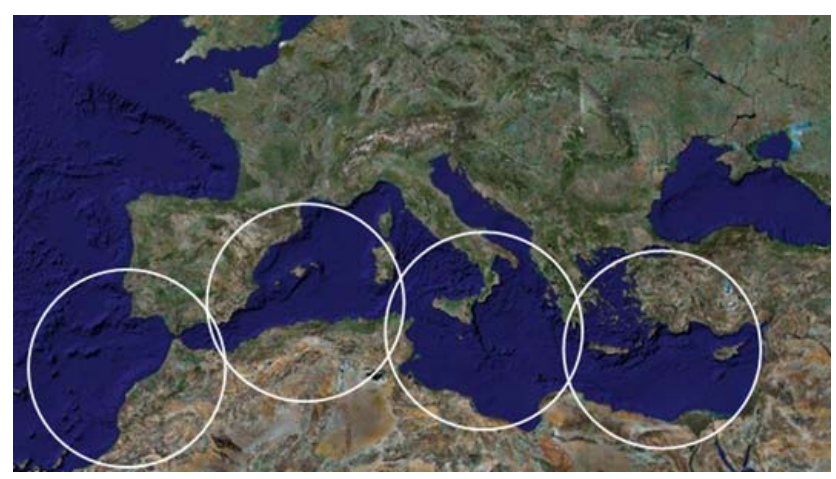

Fig. 3. Spatial coverage required for the Mediterranean Sea.

remote sensing applications, in which the target is extended, it is appropriate to define $\sigma_{0}$ (normalized radar cross section) as the radar cross section per unit area of the scene as a random variable, with a mean $\sigma_{0}$, which in general varies from one resolution element to another. The following equation expresses the average SNR of a single radar pulse viewing an extended target with homogeneous mean specific backscatter coefficient $\sigma_{0}$.

$\mathrm{SNR}=\left(\frac{P_{\mathrm{t}} G^{2} \lambda^{2}}{(4 \pi)^{3} R^{4}}\right)\left(\frac{1}{L}\right) \sigma^{0}\left(\frac{c \tau}{2 \sin (\eta)}\right)\left(\frac{\lambda R}{L_{\mathrm{a}}}\right)\left(\frac{1}{k \mathrm{NTB}}\right)$

where $P_{\mathrm{t}}$ is the transmitted power, $G$ the antenna gain, $R$ the range distance, $L_{\mathrm{a}}$ the antenna aperture, $\tau$ the pulse width, $\lambda$ the radar signal wavelength, $c$ the speed of light, $N$ the noise figure, $T$ the noise temperature, $L$ the loss, and $B$ is the Boltzmann constant.

For oceanographic applications, the normalized radar cross-section is strongly dependent on polarization (vertical $V V$ or horizontal $H H$ ), angle of incidence, local wind speed and sea-state. For our performance prediction we will use indicative values. NESTRAD is meant to detect tsunamiinduced radar cross section modulations in the largest possible area. We will therefore consider incidence angles ranging 
from $0^{\circ}$ (nadir) to $80^{\circ}-85^{\circ}$ (low grazing). For ocean observations, vertical polarization yields higher RCS than horizontal, thus driving the choice for a single polarization $V V$ system. For low grazing angles, the RCS can be taken approximately equal to $-30 \mathrm{~dB}$. For airship-borne real aperture radars, PRF (pulse repetition frequency) constraints come from Nadirtransmit interferences and range ambiguities, i.e.

$W_{\mathrm{a}}>2 \lambda R(\mathrm{PRF}) \tan (\eta) / c$

Further, if Doppler measurements are considered, the PRT (pulse repetition time) must also be reasonably shorter than the target decorrelation time, which is in the order of milliseconds.

Considering a continuous waveform (FMCW), follows an indicative system design for NESTRAD as shown in Table 1. C-band is chosen for its sensitivity to sea-surface roughness and cloud penetrating capabilities. Ka-band would also provide a very good sensitivity to ocean roughness, but unfortunately is much more affected by bad weather conditions (Danklmayer, 2009) than C-band and hence not a good choice for a reliable early warning tool. Vertical polarization is chosen given the higher RCS with respect to horizontal polarization. Beam steering capabilities allow the coverage from nadir to low grazing in elevation and from $-60^{\circ}$ to $+60^{\circ}$ from broadside in the azimuth direction, thus covering a circular sector approximately $120^{\circ}$ wide. From Table 2 we can see that in the far range a SNR of $13 \mathrm{~dB}$ can be achieved at broadside, corresponding to an acceptable $10 \mathrm{~dB}$ SNR at $60^{\circ}$ off broadside. Even though azimuth resolution in the far range appears to be poor $(2000 \mathrm{~m})$, it is still acceptable to resolve tsunami shadows, the latter having spatial extensions of thousands times tens of kilometers. This huge spatial scale will also be of great advantage for resolving the shadows, i.e. changes in the RCS of few up to $5 \mathrm{~dB}$, since averaging over large areas is possible and thus will reduce uncertainties due to local statistical variation in ocean backscatter to a large extent. Finally it has to be stated that it is not yet clear how much bad weather situations with rain, turbulent and strong winds and huge seas will affect or limit the detection capabilities of the radar. However, since the performance shown in Table 2 is based on minimum power requirements, the sensitivity of the system can be easily enhanced by increasing the power, which is absolutely no problem on airships.

\subsection{Doppler mode}

Being stationary, the system can be used as a pulse Doppler radar to estimate sea surface radial velocities. Tsunami orbital velocities appear as anomalous surface currents whose value is crucially dependent on tsunami magnitude and bathymetry. In this section, we consider the problem of tsunami orbital velocity detection with a real aperture radar at microwave frequencies.
Table 1. NESTRAD design parameters.

\begin{tabular}{|c|c|}
\hline \multicolumn{2}{|l|}{ System Parameters } \\
\hline Antenna & $\begin{array}{l}10 \mathrm{~m} \times 3 \mathrm{~m} \\
\text { phased array }\end{array}$ \\
\hline Frequency & $5 \mathrm{GHz}$ \\
\hline Polarization & VV \\
\hline Path Loss & $3 \mathrm{~dB}$ \\
\hline Noise Figure & $3 \mathrm{~dB}$ \\
\hline \multicolumn{2}{|l|}{ Antenna Parameters } \\
\hline Antenna Aperture & $30 \mathrm{~m}^{2}$ \\
\hline Antenna Gain & $51 \mathrm{dBi}$ \\
\hline Side lobe level & $-15 \mathrm{~dB}$ \\
\hline Max. scan angle from broadside (elevation) & $45^{\circ}$ \\
\hline Max. scan angle from broadside (azimuth) & $60^{\circ}$ \\
\hline
\end{tabular}

The accuracy of the velocity measurement yielded by a stationary Doppler radar system is given by the square root of the variance of the velocity estimator (Doviak et al., 1993; Rodriguez et al., 1992):

$$
\begin{aligned}
\operatorname{var}(\hat{v})= & \left(\frac{\lambda}{4 \pi \mathrm{PRF}}\right)^{2} \frac{1}{2 N^{2}}\left(\frac{1-|\rho[1]|^{2}}{|\rho[1]|^{2}}\right) \\
& \sum_{n=-(N-1)}^{(N-1)}|\rho[n]|^{2}(N-|n|)
\end{aligned}
$$

where $\hat{v}$ is the line-of-sight velocity estimator, $N$ is the number of samples, $\rho[n]$ is the correlation coefficient at PRT $\cdot n$ time lag, and $\lambda$ is the radar wavelength. Substituting the design values given in Tables 1 and 2 into the formula we obtain a velocity accuracy of approx. $1 \mathrm{~cm} / \mathrm{s}$ for a SNR of $20 \mathrm{~dB}$. This value should in principle allow the retrieval of the tsunami-induced surface current signature also in the open ocean without requiring the wave to propagate in shallow bathymetry.

Note that the correlation coefficient is not only dependent on the time lag, but also on the spatial extension of the radar resolution cell. Being a real aperture radar the quality of the Doppler measurement might be degraded in the far range with a consequent constraint on the coverage area for this operating mode, i.e. the spatial coverage will be much lower $(\sim 100 \mathrm{~km})$ than for the RCS mode. The velocity accuracy can be enhanced by increasing the PRF, and thus also a tsunami with low horizontal orbital velocities should principally be detectable, since these tsunami-induced velocity changes again apply to a very large area. However, the spatial coverage limitation emphasizes to treat this detection mode only as an add-on, which is not able to fully replace, but probably to add significant value to the RCS mode. 
Table 2. NESTRAD performance at near and far range.

\begin{tabular}{lll}
\hline Waveform Parameters & far range & near range \\
\hline Incidence angle & $70^{\circ}-80^{\circ}$ & $20^{\circ}$ \\
$\sigma_{0}$ & $-30 \mathrm{~dB}$ & $-20 \mathrm{~dB}$ \\
PRF & $800 \mathrm{~Hz}$ & $2 \mathrm{kHz}$ \\
Pulse width & $1.25 \mathrm{~ms}$ & $0.5 \mathrm{~ms}$ \\
Peak power & $100 \mathrm{~W}$ & $1 \mathrm{~W}$ \\
Bandwidth & $150 \mathrm{MHz}$ & $150 \mathrm{MHz}$ \\
Duty cycle & $100 \% \mathrm{FMCW}$ & $100 \% \mathrm{FMCW}$ \\
SNR & $13 \mathrm{~dB}$ & $40 \mathrm{~dB}$ \\
Range resolution & $1 \mathrm{~m}$ & $3 \mathrm{~m}$ \\
Azimuth resolution & $2000 \mathrm{~m}$ & $100 \mathrm{~m}$ \\
\hline
\end{tabular}

\subsection{ATI (along-track interferometry) mode and SAR (synthetic aperture radar)}

Considering the airship platform to be moving, rather than being stationary, offers additional possibilities of operating the radar. With a linear movement of the platform along the antenna aperture (azimuth) a much larger antenna aperture can be synthesized, resulting in the typical high-resolution SAR imaging mode, which is used by almost all the current remote-sensing radar systems. Thus, assuming an aperture length of $10 \mathrm{~m}$ and a signal bandwidth of $150 \mathrm{MHz}$, the spatial resolution would be around $5 \mathrm{~m}$ in azimuth (flight direction) and $1 \mathrm{~m}$ in range (radar look direction), independent of the sensor height above ground. The SAR mode, however, is not a good candidate for ocean monitoring, since it requires coherent summation of radar signals over the whole length of the synthetic aperture. At the low speed of an airship $(\sim 50 \mathrm{~m} / \mathrm{s})$, building up the synthetic aperture requires acquisition time in the order of seconds, whereas the correlation length of ocean wave movement is in the order of milliseconds. SAR imaging, in this case, makes only sense over solid earth targets, which stay coherent over longer periods of time.

Coming back to tsunami detection, the movement of the platform also opens up the opportunity of using along-track interferometry (ATI) to measure velocities of ground targets and thus also orbital velocities of ocean waves. The principal of ATI is to use two antennas at slightly different positions in the along-track (flight-) direction. If a target is moving in the radial direction (radar look direction), the Doppler spectra at the two antennas will be different and, with this difference, the radial velocity component can be estimated. The details of ATI and ground moving target indication (GMTI) can be found in (Sharma, 2004). With regard to tsunami detection it is particularly important to know, whether the expected orbital velocities can be resolved by ATI techniques or not. The minimal resolvable radial target velocity $v_{\text {min }}$ depends on the velocity of the platform $v_{\mathrm{P}}$, the radar wave- length $\lambda$ and the length of the antenna aperture $L$. In order to generate two separate antennas in along-track with the actual NESTRAD design, the $10 \mathrm{~m}$ phased array antenna can be electronically divided into two smaller antennas with the length of $5 \mathrm{~m}$ each, which would then yield a minimal resolvable velocity of

$v_{\min }=\frac{\lambda v_{\mathrm{P}}}{2 L}=0.025 \frac{\mathrm{m}}{\mathrm{s}}=2.5 \frac{\mathrm{cm}}{\mathrm{s}}$,

assuming a platform velocity of $50 \mathrm{~m} / \mathrm{s}$. Since $2.5 \mathrm{~cm} / \mathrm{s}$ is in the range of a large tsunami, the accuracy would have to be increased for a small tsunami, e.g. by increasing the aperture $L$. Unfortunately this is not the only limiting factor for ATI over the ocean. In order to enable sufficiently coherent data for resolving the required ocean currents, the time lag between the two ATI acquisitions has to be in the order of the decorrelation time, i.e. milliseconds. The distance $d$ of the two individual antenna phase centers with respect to correlation time $\tau_{\text {corr }}$ and platform velocity $v_{\mathrm{P}}$ is given by (Romeiser, 2000)

$d=\tau_{\text {corr }} \cdot v_{\mathrm{P}}$.

As for the aforementioned configuration and a correlation time of $1 \mathrm{~ms}$, the distance $d$ would have to be $5 \mathrm{~cm}$, which cannot be achieved by using only one electronically divided antenna. Hence the biggest problem and limiting factor is the low platform velocity, which cannot be significantly increased. Only expensive solutions using at least two very large antennas are able to ensure sufficient ATI performance for tsunami detection.

Interferometry (InSAR), polarimetry (PolSAR), polarimetric SAR interferometry (PolInSAR) and also bistatic geometries (e.g. using two NESTRAD platforms) are further options of using the radar.

\section{NESTRAD's multi-purpose approach}

Since a tsunami occurs only rarely, a sensor like NESTRAD would be too expensive and inefficient if designed exclusively for the purpose of tsunami warning. As already pointed out in the previous sections there are many different possibilities and options/modes to operate an advanced radar instrument. Due to the fact that radar instruments nowadays can be very lightweight (easily below $50 \mathrm{~kg}$ if required), and that airships are able to carry huge payloads up to a few tons, there is enough space and mass budget left for arbitrary additional sensors (e.g. optical, GNSS, infrared, more radar, etc.) on the same platform, which could be used for many applications. Beyond the capability of quick and effective tsunami detection, such a system could be able to serve for example for sea state monitoring in general, ship tracking (traffic, piracy, etc.), reconnaissance and surveillance, local weather monitoring, hurricane monitoring, volcano monitoring, flood monitoring, traffic monitoring, and as a communication relay station. 


\section{Conclusions}

In this paper, a concept for near-field tsunami early warning is presented. NESTRAD consists of a phased array antenna accommodated inside a stationary or slowly moving airship meant to provide continuous monitoring of the ocean surface. A preliminary performance prediction is given, and the system appears to be capable of providing off-shore measurements of tsunami-related features.

The initial review of tsunami physical features has highlighted a plethora of phenomena that have received widespread attention in the remote sensing literature. Research in the field was mainly substantiated by data from LEO satellites. However, unless an inter-linked constellation of a large number $(>50)$ of satellites is considered, LEO satellites cannot match the challenging requirements posed by near-field tsunami early warning. All possible space platforms have then been considered and stratospheric airships were found to be able to serve as ideal platforms for continuous monitoring of large ocean areas.

Tsunami shadows have been found to be the most promising tsunami related signature for detection using radar sensors, since the observed RCS modulations of a few $\mathrm{dB}$ over very large areas should be easy to resolve even using very minimalistic radar designs. The RCS mode also provides a huge permanent coverage of approx. $1000 \mathrm{~km}$ disk diameter from $20 \mathrm{~km}$ platform height.

The physical characterization of tsunami waves from a radar remote sensing viewpoint is still in its initial stages, and more information is needed for a robust characterization. Open questions are, among others, related to information on the initial stages of the wave, e.g.: how long does it take for orbital velocities and/or for tsunami shadows to build up to a maximum after the initial generation? Which is the dependence of tsunami shadows on atmospheric weather? Which is the dependence of tsunami shadows on tsunami magnitude?

Finally we shall like to point out that first experimental tests using radar on an airship platform are planned by the Microwaves and Radar Institute of DLR in cooperation with the University of Jülich (Germany) and the Zeppelin company for the year 2011.

Acknowledgements. The presented work is part of the GITEWS project (German Indonesian Tsunami Early Warning System) funded by the German Federal Ministry for Education and Research (BMBF), Grant 03TSU01.

Edited by: J. Lauterjung

Reviewed by: two anonymous referees

\section{References}

Afullo, T. J. and Odedina, P. K.: On the K-factor distribution and diffraction fading for southern Africa, Trans. SAIEE, 97(2), 172$181,2006$.

Allen, T.: The Story of GANDER, Sensors 2006, 6(3), 249-259, doi:10.3390/s6030249, 2006.

Anderson, S. J.: Prospects for Tsunami Detection and Characterisation with HF Skywave Radar, Proc. IEEE International Radar Conference Radar 2008, Adelaide, Australia, 666-672, 2008.

Bryant, E.: Tsunami: the underrated hazard, Cambridge University Press, New York, 2001.

Cooney, M.: Colossal spy airships with 15 -story radars get $\$ 400 \mathrm{M}$ reality check, available at: www.networkworld.com/community/ node/41368 (last access: September 2010), 2009.

Danklmayer, A. and Chandra, M.: Precipitation Effects for Ka-band SAR, Proc. Advanced RF Sensors For Earth Observation (ARSI), Noordwijk, 2009.

Doviak, R. J. and Zrnic, D. S.: Doppler Radar and Weather Observations, 2nd ed., San Diego, CA: Academic Press, 1993.

Godin, O. A.: Air-sea interaction and feasibility of tsunami detection in the open ocean, J. Geophys. Res., 109, C05002, doi:10.1029/2003JC002030, 2004.

Hanlon, M.: Stratospheric airship reaches near-space altitude during demonstration flight, available at: www.gizmag.com/go/ 4901/ (last access: September 2010), 2005.

Henderson, F. M. and Lewis, A. J. (eds.): Principles and applications of imaging radar, Manual of remote sensing: Third edition, Volume 2, John Wiley and Sons, Inc., Somerset, NJ (United States), ISBN 0-471-33046-9, 1998.

Krieger, G., Gebert, N., Younis, M., Bordoni, F., Patyuchenko, A., and Moreira, A.: Advanced concepts for ultra-wide swath SAR imaging, Proc. European Conference on Synthetic Aperture Radar EUSAR 2008, Friedrichshafen, Germany, 2008.

Lautrup, B.: Tsunami Physics, Science Journal Kvant, 22-25, 2005.

Lipa, B. J., Barrick, D. E., Bourg, J., and Nyden, B. B.: HF Radar Detection of Tsunamis, J. Oceanography, 62, 705-716, 2006.

Modica, G. D., Nehrkorn, T., and Myers, T.: An Investigation of Stratospheric Winds in Support of the High Altitude Airship, available at: ams.confex.com/ams/pdfpapers/128256.pdf (last access: September 2010), 2007.

Myers, R. G.: Potential For Tsunami Detection And Early-Warning Using Space-Based Passive Microwave Radiometry, Master Thesis at the Massachusetts Institute of Technology, available at: dspace.mit.edu/bitstream/handle/1721.1/42913/244303119. pdf?sequence=1 (last access: September 2010), 2008.

Okal, E. A.: Tsunami detection by satellite altimetry, J. Geophys. Res., 104(B1), 589-615, 1999.

Perkins, S.: Catching waves: ocean-surface changes may mark tsunamis, Science News, vol. 165, February 21, p. 116, 2004.

Qureshi, I.: An airship that can counter cross-border terrorism, available at: spot.colorado.edu/ $\sim$ dziadeck/airship/india. htm, 2003.

Rodriguez, E. and Martin, J. M.: Theory and design of interferometric synthetic aperture radars, Radar and Signal Processing, IEE Proceedings F, 139(2), 147-159, 1992.

Romeiser, R. and Thompson, D. R.: Numerical Study on the AlongTrack Interferometric Radar Imaging Mechanism of Oceanic Surface Currents, IEEE T. Geosci. Remote, 38(1), 446-458, 2000 . 
Santek, D. A. and Winguth, A.: A satellite view of internal waves induced by the Indian Ocean tsunami, Int. J. Remote Sens., 28(13-14), 2927-2936, ISSN:0143-1161, 2007.

Sharma, J.: The Influence of Target Acceleration on Dual-Channel SAR-GMTI (Synthetic Aperture Radar Ground Moving Target Indication) Data, Master thesis, Department of Geomatics Engineering, Calgary, Alberta, available at: www.ucalgary.ca/ engo_webdocs/MJC/04.20206.jsharma.pdf (last access: September 2010), 2004.
Stosius, R., Beyerle, G., Helm, A., Hoechner, A., and Wickert, J.: Simulation of space-borne tsunami detection using GNSSReflectometry applied to tsunamis in the Indian Ocean, Nat. Hazards Earth Syst. Sci., 10, 1359-1372, doi:10.5194/nhess-101359-2010, 2010.

Troitskaya, Y. I. and Ermakov, S. A.: Manifestations of the Indian Ocean tsunami of 2004 in satellite nadir-viewing radar backscatter variations, Int. J. Remote Sens., 29(21), 6361-6371, 2008. 